\title{
The X-ray Astronomy Satellite ASTRO-H
}

\author{
Manabu Ishida* \\ ISAS/JAXA \\ E-mail: ishidadastro.isas.jaxa.jp
}

\begin{abstract}
$A S T R O-H$ is the 6th Japanese X-ray astronomy satellite having been developed under a vast collaboration of the international X-ray astronomy community. The Soft X-ray Spectrometer (SXS) onboard ASTRO- $H$ can realize fine X-ray spectroscopy with energy resolution of more than 20 times as good as the standard X-ray detector CCD. Since the SXS is a non-dispersive detector, it works not only for point sources but also for diffuse X-ray sources. The advent of the SXS is believed to open up a new era in the field of X-ray spectroscopy. The other main instrument, the hard X-ray imager, will have a larger effective area than NuSTAR, although spatial resolution is somewhat limited. The integration and all the tests of ASTRO- $H$ spacecraft has already been finished. The spacecraft is ready to be shipped to the launch site: Tanegashima Space Center. After the launch site operation to be started in early December 2015, ASTRO- $H$ will be launched by the end of 2016 March.
\end{abstract}

The Golden Age of Cataclysmic Variables and Related Objects - III

7-12 September 2015

Palermo, Italy

* Speaker.

${ }^{\dagger}$ A footnote may follow. 


\section{ASTRO-H Mission}

ASTRO-H is the 6th Japanese X-ray astronomy satellite, following Hakucho (1979-1985), Tenma (1983-1985), Ginga (1987-1991), ASCA (1993-2000), and Suzaku (2005-2015). It will be launched with the H-2A rocket from Tanegashima Space Center Japan into a circular orbit with an altitude of $575 \mathrm{~km}$. The inclination of the orbit is $31^{\circ}$, and the orbital period is $96 \mathrm{~min}$. The launch will be carried our by the end of JFY2015 (= March 2016). The ASTRO-H project has been executed through a vast international collaboration. Significant contribution has been made to the project by more than 200 scientists from Japan, US, Netherland, Swiss, Ireland, UK, France, Canada. Four major space institutes, JAXA, NASA, ESA, and CSA have also supported the project. The readers are advised to refer to [1] for full detail of ASTRO-H.

\section{Scientific Instruments}

\subsection{Overview}

In Fig. 1 introduced are the four scientific instruments with horizontal strips indicating the energy bands they cover. Their brief profiles are as follows.

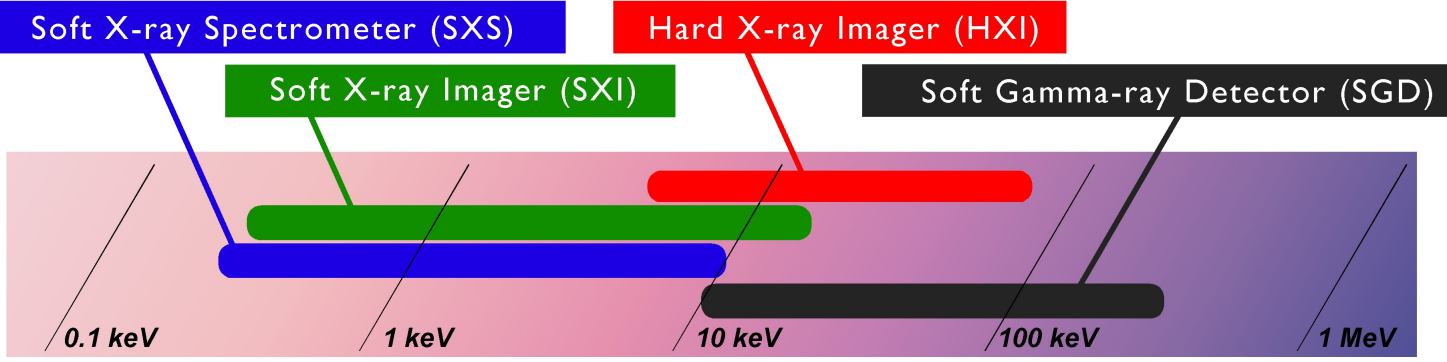

Figure 1: Detector systems of $A S T R O-H$ and the energy bands they cover.

- Soft X-ray Spectrometer (SXS) $\cdots$ One of the two main instruments of ASTRO-H. The detector which is made of mercury telluride ( $\mathrm{HgTe}$ ) is cooled down to $\sim 50 \mathrm{mK}$ to detect $\mathrm{X}$ ray as temperature enhancement of the detector ([2]). Energy resolution is $<7 \mathrm{eV}$ at the $\mathrm{X}$-ray energy $6 \mathrm{keV}$, which is more than 20 times as good as the standard X-ray detector CCD. This is the first non-dispersive detector system that has much better energy resolution than the $\mathrm{CCD}$, and moreover, has better energy resolution than any other grating spectrometer now at work in orbit above $\sim 1 \mathrm{keV}$. The advent of the SXS is believed to bring about a breakthrough in the field of X-ray spectroscopy. The SXS is equipped on the focal plane of one of the two soft X-ray telescopes (SXT-S, [3]).

- Hard X-ray Imager (HXI) $\cdots$ The other main instrument of ASTRO-H that covers the hard X-ray band 5-78 keV with multi-layered $\mathrm{Si}$ and CdTe detectors (44). There are two identical HXI modules on ASTRO-H. The hard X-rays are collected to the HXI detectors with two identical hard X-ray telescopes (HXTs, [5]). A similar type imaging system is already realized by NuSTAR launched in 2013 (鸟), yet the HXI system has a larger effective area (see below and Fig. 3). 
- Soft X-ray Imager (SXI) … A standard CCD camera but with quite a large field of view, a square with $\sim 40$ arcmin on a side ([7]). The SXI is located on the focal plane of the other soft X-ray telescope (SXT-I, [3]). Note that the design of the two SXT modules is completely identical.

- Soft $\gamma$-ray Detector (SGD) … SGD is the only detector system that has no focusing mirror. It is composed of multi-layered semi-conductor plates, and enhances sensitivity by applying the Compton camera principle to events detected by the plates ([9).

Of them profiles of the two main instruments are described in more detail in the following subsections.

\subsection{Soft X-ray Spectrometer (SXS)}

The SXS detects an X-ray photon as temperature increase. In the low temperature limit, heat capacity of a solid body is proportional to $T^{3}$. Hence it is necessary to cool the detector to get a larger pulse height against a given X-ray energy. Since the energy of a single X-ray photon is tiny, however, it is necessary to cool the detector down to an ultimately low temperature. In the case of the SXS, the detector part is deeply embedded in a vacuum adiabatic shield (Dewar) and is cooled to some $50 \mathrm{mK}$ by means of an adiabatic demagnetization refrigerator (ADR) and a series of mechanical coolers.

The X-ray absorber is made of mercury telluride (HgTe) whose pixel size is $818 \mu \mathrm{m}$. The pixel array comprises of $6 \times 6$ format with a pixel pitch of $832 \mu \mathrm{m}$. Since the focal length of the SXT-S is $5.6 \mathrm{~m}$, the field of view of the SXS is a square with 3.06 arcmin on a side. Figure 2 shows an energy spectrum of the flight detector array being illuminated by a series of fluorescent targets ([1]). The energy resolution evaluated by co-adding data from all 36 pixels is $4.7 \mathrm{eV}$ for ${ }^{55} \mathrm{Mn}$ $5.9 \mathrm{keV}$ X-ray. See [2] and [3] for more detail about the SXS and the SXT, respectively.

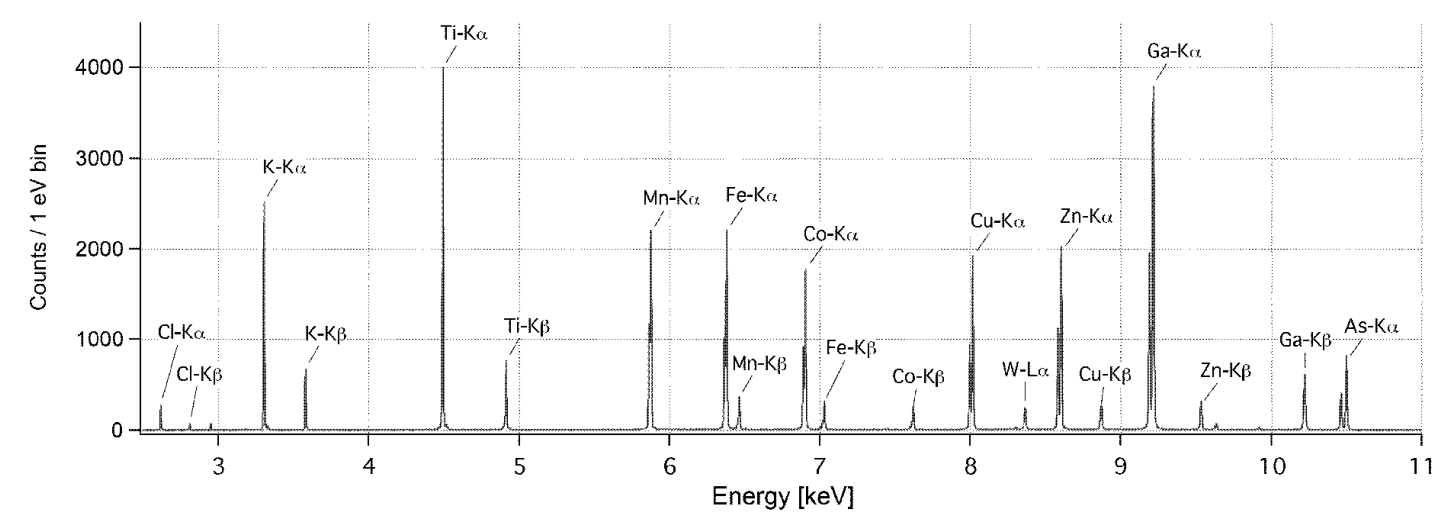

Figure 2: The SXS spectrum taken with a series of fluorescent X-ray sources. 112

\subsection{Hard X-ray Imager (HXI)}

The HXI detector comprises of four layers of double-sided silicon strip detectors (DSSD) and a single layer of CdTe imaging detector whose thickness is $0.5 \mathrm{~mm}$ and $0.75 \mathrm{~mm}$, respectively [1] 4]. 
Two modules of the HXI system are onboard ASTRO-H, which are referred to as HXI-1 and HXI-2. They are located on the focal plane of the two independent hard X-ray telescope modules HXT-1 and HXT-2 ([5]) whose focal length are $12 \mathrm{~m}$. The pitch of the strips of the HXI detector is $0.25 \mathrm{~mm}$ which corresponds to $\sim 4$ arcsec, given the HXT's focal length of $12 \mathrm{~m}$. Reflectors of the HXTs are produced by utilizing the Pt/C multi-layer supermirror technology [8], enhancing reflectivity over $10 \mathrm{keV}$ up to the Pt K-edge energy $78 \mathrm{keV}$. With this combination of the detector and the telescope, the HXI system has a large effective area in the band 5-78 keV. In Fig. 3 3 shown is the effective area of the HXI system (one module) in comparison to that of NuSTAR (【6). NuSTAR adopts hard X-ray

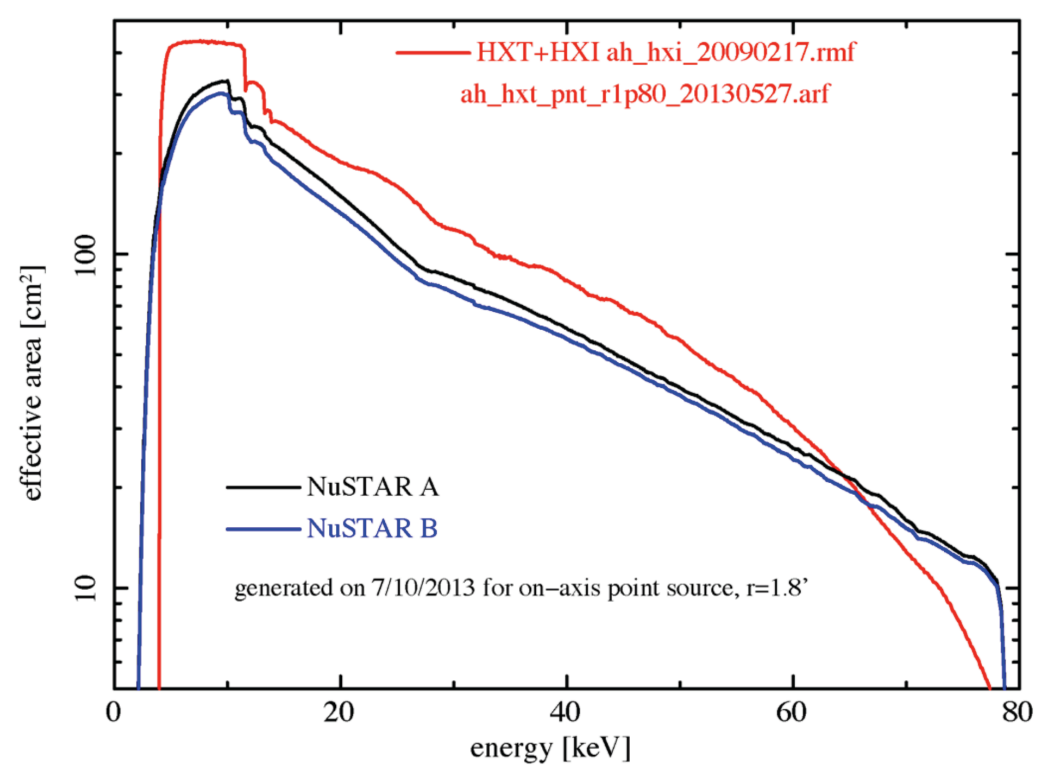

Figure 3: Effective area of the HXI system (one module) in comparison with that of NuSTAR ([6])

imaging system that is quite similar to ASTRO-H. Although the imaging capability of the NuSTAR mirror $\sim 50$ arcsec is significantly better than that of ASTRO-H (1.9 $\operatorname{arcmin}$ at $30 \mathrm{keV}$ : 【5]), the effective photon-collecting area of the telescope is somewhat larger in ASTRO-H. This is mainly because the longer focal length of the ASTRO-H telescope $(12 \mathrm{~m})$ than that of NuSTAR $(10.4 \mathrm{~m})$.

\section{Project History}

In Fig. 4 4 shown is a diagram describing the history of the ASTRO- $H$ project. The first proposal was submitted to ISAS/JAXA in 2003 November. This is just after the three Japanese space agencies (ISAS, NASDA, and NAL) are combined into one agency named JAXA. Although this proposal was not successful, a refined version was completed and resubmitted by ISAS in 2005 September. This was just after the launch of Suzaku (2005 July 10th). This second version was finally approved by JAXA in 2007. After some basic studies on feasibility, the project started on 2008 October 1st. After $\sim 1.5$-yrs study, the project progress was examined in the preliminary design review (PDR) in 2010 May, and the project team is allowed to start designing the flight model (FM). 


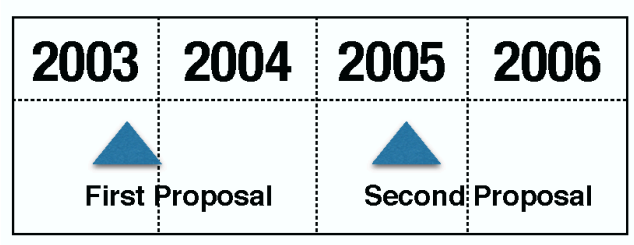

2003 First Proposal (NeXT)

2005 Second Proposal (NeXT)

2008 JAXA ASTRO-H Project started

2010 Preliminary Design Review (PDR)

2012 Detail Design Review (CDR)

2014 1st Integration test completed (EIC/MIC)

2014 Oct. CDR2

2014 Nov. Final I\&T started

2015 July Thermal-Vac. test completed

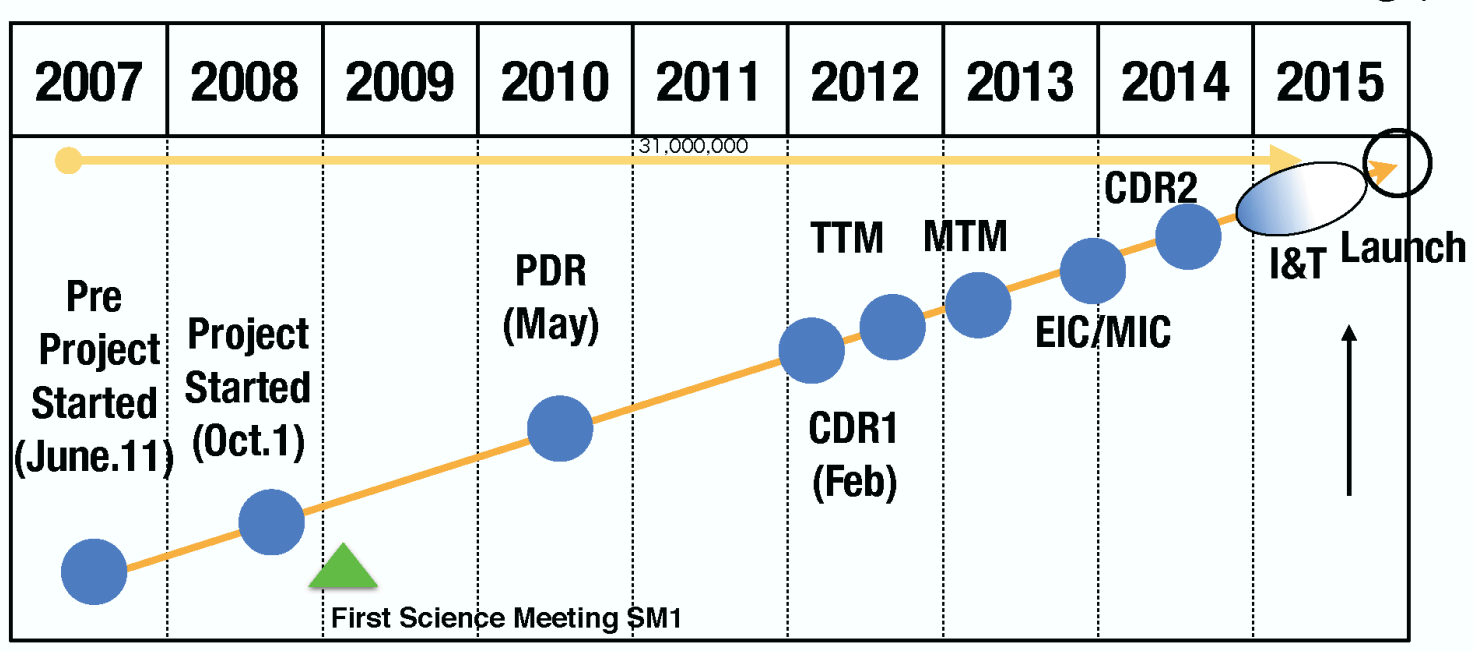

Figure 4: History of the $A S T R O-H$ project.

In 2012 February, the project team had the critical design review (CDR), and was allowed to manufacture the FM payloads. However, part of requirements to the payloads cannot be finalized at this moment. They are mainly mechanical loads at the time of launch, and thermal condition in orbit. We therefore started the FM production by assuming plausible specifications on them, and afterward they were verified in the TTM (thermal test model) test and MTM (mechanical test model) test carried out during 2012-2013 time frame. After validation of the mechanical loads and the thermal condition with these tests, we started electric and mechanical interface check using the FM in 2013-2014. After confirming everything is fine, we carried out the FM integration and associated tests, which started in 2014 November and continued just for one year.

\section{Recent Project Status}

The project status since 2015 April is summarized in Fig. 5 Fabrication of the ASTRO-H FM started on April 1st this year. The SXS Dewar was first assembled onto the base structure of the satellite, followed by the SXI integration and then the optical bench. The entire ASTRO-H spacecraft is basically an X-ray telescope. Accordingly, its interior should be a cavity for X-ray photons collected by the X-ray mirrors to pass through and to arrive at the detectors without any interference. Because of this, all electronic equipments are mounted on the side panels of the spacecraft. In parallel to the fabrication of the detectors and the optical bench, electronic tests of the electronic equipments had been carried out. On May 21-22, the first full functional test was 


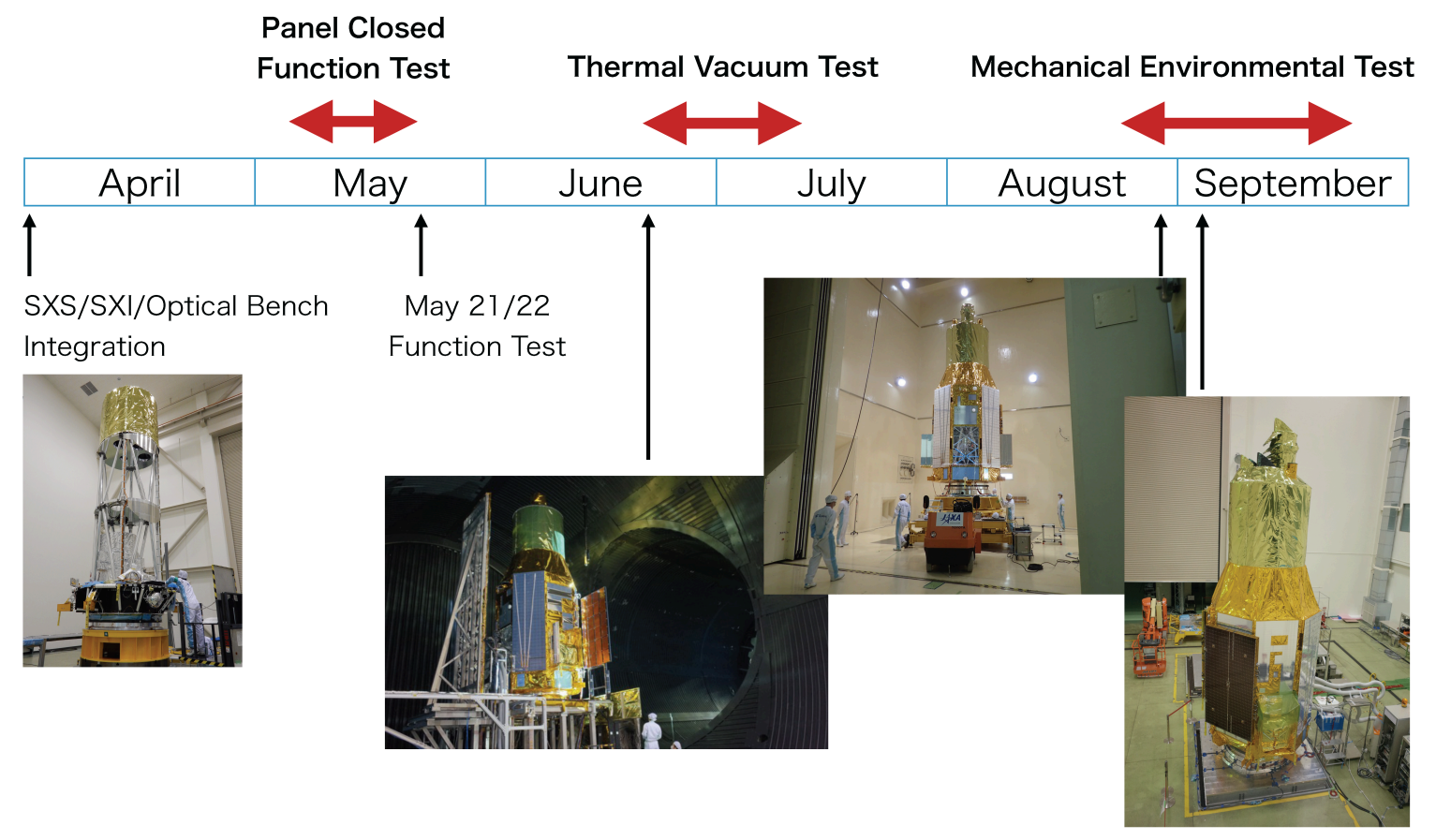

Figure 5: The activity of the ASTRO-H project since 2015 April.

carried out in the configuration of all the side panels being closed. The side panels have never been removed since this epoch.

Thermal vacuum test and mechanical environmental test were carried out in June-July and August-September, respectively. The former test aims at verifying validity of the thermal mathematical models of the payloads and the entire satellite system as well, and confirming proper performance of the payloads in the space environment. The latter test, on the other hand, was made to see if the spacecraft survives the mechanical load (acoustic, mechanical shock and vibration) at the time of launch without losing any functionality. Fortunately, the spacecraft and the payloads all pass these tests. At the end of November 2015, the spacecraft is ready for shipment toward the launch site, Tanegashima Space Center (TNSC). We will start the launch site operation in early December 2015. The ASTRO-H spacecraft will be launched by the end of the Japanese fiscal year 2015 (by the end of 2016 March).

\section{Schedule after the Launch}

After the launch, we define the following phases.

- Initial checkout phase … First 6 weeks (1.5 months) after the launch. Of them, the first one week is referred to as the initial critical phase in which deployment of the solar array paddles, establishment of functionality of the attitude controlling system, start-up of the SXS refrigerators, and deployment of the extensible optical bench to realize the $12 \mathrm{~m}$ focal length of the HXTs will be carried out. The following 5 weeks are dedicated to confirmation of the bus system functionality and start-up of the other scientific instruments. 
- Performance verification (PV) phase $\cdots$ After the initial checkout phase, celestial objects which were selected by the ASTRO-H science working group will be observed. This phase continues for 6 months. The purpose of this phase is to demonstrate capability of ASTRO-H to scientists worldwide. $90 \%$ of the time is used for observations of the SWG targets, and the remaining $10 \%$ is the observatory time handled by the PI/project manager.

- Guest observer (GO) phase $\cdots$ After the PV phase, the GO phase will start. Most of the time will be opened to guest observers. Like the case of Suzaku, we will solicit observation proposals from Japan, US, and Europe separately. Percentage of time allocation among them will be decided according to contribution of each party.

\section{References}

[1] Takahashi, T., Mitsuda, K., Kelley, R., et al. 2014, SPIE, 9144, 25

[2] Mitsuda, K., Kelley, R. L., Akamatsu, H., et al. 2014, SPIE, 9144, 2A

[3] Soong, Y., Okajima, T., Serlemitsos, P. J., et al. 2014, SPIE, 9144, 28

[4] Sato, G., Kokubun, M., Nakazawa, K., et al. 2014, SPIE, 9144, 27

[5] Awaki, H., Kunieda, H., Ishida, M., et al. 2014, Applied Optics, 53, 7664

[6] Harrison, F. A., Craig, W. W., Christensen, F. E., et al. 2013, Ap. J., 770, 103

[7] Hayashida, K., Tsunemi, H., Tsuru, T. G., et al. 2014, SPIE, 9144, 29

[8] Ogasaka, Y., Tamura, K., Shibata, R., et al. 2006, SPIE, 6266, 150

[9] Fukazawa, Y., Tajima, H., Watanabe, S., et al. 2014, SPIE, 9144, 2C

\section{DISCUSSION}

DMITRY BISIKALO: What is the expected life time of the Astro-H ?

MANABU ISHIDA: The spacecraft system is designed to work for three years in orbit. The SXS uses liquid ${ }^{3} \mathrm{He}$ as a coolant which can also last for three years. However, the temperature of the SXS detector can be kept at $50 \mathrm{mK}$ even after all ${ }^{3} \mathrm{He}$ is evaporated.

HANNES BREYTENBACK: What time-resolution will Astro-H SXT/HXT be able to achieve ?

KOJI MUKAI: As for the SXS, some $60 \mathrm{msec}$ is possible.

KENJI TANABE: The CCD camera you are going to equip on the telescope is actually the same or different from the point of the sensitivity? Is it possible to detect X-ray photon directly?

MANABU ISHIDA: The sensitivity is different. Thickness of the depletion layer for optical CCD is in general $\sim 5 \mu \mathrm{m}$. This is not enough to stop X-rays. Recent CCDs used for X-ray observation have the depletion layer thickness of more than $100 \mu \mathrm{m}$ in general. 


\section{Note added in proof}

The ASTRO-H satellite was launched on 2016 February 17th 17:45 (JST) from Tanegashima Space Center with the 30th H-2A rocket of JAXA, and renamed Hitomi (implying a 'pupil', aiming at looking into new blackholes and deep universe). The launch operation was successful and the spacecraft was thrown into the planned low-earth orbit with an altitude of $575 \mathrm{~km}$. Although all the science instruments (SXS, SXI, HXI and SGD) were perfectly started up, there was a malfunction in the attitude and orbit controlling system, and we permanently lost contact with ASTRO-H on March 26th, 2016. Nevertheless, the science team has been struggling to extract new science from the data taken during the initial $\sim 1$ month, and one Nature paper on the Perseus cluster was already published. The team has gratually recovered from this tragedy, and has been working hard for a recovery mission, which carries a Soft X-ray Spectrometer, equivalent to the SXS onboard ASTRO$H$, again. 